\title{
A Review on Helmet Detection by using Image Processing and Convolutional Neural Networks
}

\author{
Prajwal M. J. \\ JSS Science and Technology \\ University \\ Department of Electronics and \\ Communication Engineering \\ Mysuru-06
}

\author{
Tejas K. B. \\ JSS Science and Technology \\ University \\ Department of Electronics and \\ Communication Engineering \\ Mysuru-06
}

\author{
Varshad V. \\ JSS Science and Technology \\ University \\ Department of Electronics and \\ Communication Engineering \\ Mysuru-06
}

\author{
Mahesh Madivalappa Murgod \\ JSS Science and Technology University \\ Department of Electronics and Communication \\ Engineering \\ Mysuru-06
}

\author{
Shashidhar R. \\ JSS Science and Technology University \\ Department of Electronics and Communication \\ Engineering \\ Mysuru-06
}

\begin{abstract}
The main application of helmet detection is in traffic roads where accidents are more. Even though various measures are taken by government, it is not followed correctly by the motorcyclists, so several smart techniques should be employed. Construction industry and power substation suffer a lot of fatalities because of negligence in wearing safety helmets, hence there is a need of surveillance system that is capable of detecting helmets and preventing the deaths. A more sophisticated computer vision model that encompasses image processing, machine learning, Convolutional neural networks $(\mathrm{CNN})$, classifiers such as support vector machine (SVM), ViBe background modeling algorithm, a relevant data set containing helmets; caps; Histogram of Oriented Gradients(HOG) features and number of other techniques would solve the problem. Methods like COCO model, HOG descriptors, Hough transforms are used to detect objects. Optical character recognition(OCR), Haar like feature is used to detect number plates.
\end{abstract}

\section{General Terms}

Object classification, Helmet detection, license plate extraction.

\section{Keywords}

OCR, SVM, HOG, LBP, CNN

\section{INTRODUCTION}

India with 1.3 billion population has millions of motor vehicles running on the roads. Violation of traffic rules has become a common phenomenon, but consequence of which is unbearable. According to a report by NDTV around 400 motor cyclists perish every day in India because of not wearing helmet. If we use helmets it could increase the chances of survival by 42 percent. It also reduces the injuries upto 70 percent. As a citizen of a country one has to obey law and order and follow the same in absolute manner. But seriousness among us is not that great. One has to read a WHO report titled as "why are helmets needed?" in this report there is a comprehensive description about what happens to head if one encounters with an accident and how exactly helmet reduces the impact.

When a two wheeler meets with an accident, because of sudden deceleration the rider is thrown away from the vehicle.
If head strikes any object the motion of the head becomes zero, but with its own mass brain continues to be in motion until the object hits the inner part of the skull. Sometimes this type of head injury may be fatal in nature. In such times helmet acts as life saviour. Helmet reduces the chances of skull getting decelerated and hence sets the motion of the head to almost zero. Cushion inside the helmet absorbs the impact of collision and as time passes head comes to a halt. It also spreads the impact to a larger area, thus safeguarding the head from severe injuries. More importantly it acts as a mechanical barrier between head and object to which the rider came into contact.

In India migrant labourers working at construction sites, mining of natural resources lose their lives by not following safety measures such as wearing helmets. Daily hundreds of workers perish in this particular sector; hence there is a need of surveillance system that is capable of detecting helmets and preventing the deaths. So, in order to overcome, this smarter method has to be created. Sophisticated methods have to be built so as to overcome these problems. One way of solving this problem is smart helmet which has inbuilt sensors. More often these inbuilt sensors are multipurpose and flexible in nature. A supportive mechanism in which engine starts only when the helmet is properly worn by the person. The feasibility of this method is very low and it's quite tedious. Manual surveillance of traffic is a basic solution wherein 'on road CCTV cameras' could be used in attaining the objective of helmet detection. With ever increasing vehicle number, metro cities like Bengaluru, Delhi NCR, etc. cannot afford this manual method because of high congestion. And this also needs lot of human resource. To effectively address all these problems, we propose an efficient and a comprehensive approach for automatic helmet detection using image processing and Convolution neural network.

\section{METHODOLOGIES}

To extricate the foreground mask of the motorcycle, background subtraction and connected components labelling is used. For the stratification of riders with helmet and without helmet, SVM classifier with a linear kernel is used. Grey level histograms are used for the classification of estimated helmet region [22].Moving object detection is done using background subtraction and then K-Nearest Neighbour(KNN) classifier is used to extract the objects in motion and classify them as a 
motorcycle or non-motorcycle depending on the attributes withdrawn from their region properties and the features that are considered here are based on average hues, average intensity and circularity of each head quadrants. The proposed system helps in classifying the person wearing a helmet or not using KNN based on attributes obtained from 4 sections of segmented head region [1].

In this research article, the proposed system can be analyzed in 2 steps. In the first step the images are classified and segmented and is aimed to identify the objects in motion. To achieve this CL (Cross Line) is defined by the user and later the system classifies whether the detected system is a motorcycle or not based on the features extracted using LBP (Local Binary Pattern). In the next step the helmet recognition part is considered, here methods like hybrid descriptor is used to take out image attributes and from that SVM classifier is used to specify whether the image of interest is a helmet or a non-helmet image [2].

Moving objects segmentation is done to evaluate only the objects of interest in the image. Then descriptors were used to extract image attributes. This set of info extracted is called feature vector. By using the feature vector classifiers like multilayer Perceptron classification of images are done to know whether the captured image is a motorcycle or not. After motor vehicle detection the next step is helmet detection which is achieved in 4 steps namely, RoI extraction, Subwindow computation, attributes extraction, Image categorization. RoI is mainly used for helmet search using classifiers and next sub-window calculation is done so that only the head part is concentrated to decide whether the person is wearing the helmet or not [3].

In this research article recognition of moving vehicles is achieved using background subtraction techniques and using classifiers classification of motorcycle and non-motorcycle is done. Then Circular Hough Transform is applied on region of Interest (ROI) and in the top area if a circular arc exists, then helmet is recognized, else it is not detected [5].Motorcycle detection is carried out on the basis of tire and helmet color properties. The methodology involved here is the combination of shape, color and edge attributes to deduce motorcycle position in a video frame. Using HOG features of the motorcycle database present a classifier like SVM is used to train the model and predict whether the image is a motorcycle or not [11]

The analytical attributes are extracted using LBP and GLCM. Using the combination of GLCM analytical attributes and BP artificial neural network helmet recognition is done [8]. They have mainly used computer perception and ML expertise for automatic helmet recognition. HOG (Histogram of Gradient) algorithm is used for human detection and Circular Hough Transform for Helmet detection of specified colours only [10].

Background subtraction method is used to distinct the motion objects from static objects such as trees, roads and buildings. Local Binary Patterns [LBP], Histogram of Oriented Gradients [HOG], Scale Invariant Feature Transform [SIFT] and are used for object detection [17][18]

To detect between without helmet and full helmet Haar like features is used. The AdaBoost learning algorithm is used to boost the classification performance of a simple learning algorithm. The Hough transform is mainly applied to notice the presence of a circular shape (helmet) in a given image [19]
Smart helmet is designed for accident detection and notification using IoT notion. To achieve this they have mainly used Microcontrollers and accelerometers. The system contains a method which ensures that the riders wear the helmet without which the vehicle would not start. It is achieved through a line-up of collision switches which are fixed in the helmet which gives a communication message to microcontroller indicating that rider has not worn his helmet as a result the microcontroller switches of the engine in case the rider removes his helmet while driving. For accident detection a gyroscope and 6-axis accelerometer is used to detect angle and acceleration values along $\mathrm{z}, \mathrm{x}, \mathrm{y}$ negative, positive axis respectively. To communicate with web server a GSM module is used [4].

Adaptive background subtraction method is used on video frames to detect travelling objects. Using this result a CNN classifier is used to recognize motorcycle riders among the travelling objects. Another convolution neural network classifier is applied on RoI for identification of motorcycle riders riding without wearing a helmet [6]

Background subtraction method is used to detect moving vehicles. Motorcycle classification can be done by first using descriptors like LBP, HAAR to detect RoI and then using any one of the classifiers like SVM, multi-layer Perceptron etc. later the system is trained using PCA and other ML expertise for recognition of helmet. [9].

In this research article, workers without safety helmet are noticed in power substations. Here ViBe background model is used for background subtraction. The main objective of this model is to convert moving object segmentation into pixel classification problem. SVM is used to classify between the human and other moving object .To model local shape and appearance of the workers, Dalal and Triggs approach using dense histogram of oriented gradients (HOG) is used. It is easy to detect helmets using color feature recognition because only specific colored helmets are used in power substations [12].

In this study non helmet riders are detected and their license plate is extracted. They have used 2 YOLOv2 models to detect helmeted and non-helmeted riders. This model has the capability to detect all classes of COCO dataset. In the first set, person is detected instead of motorcycle. The second YOLOv2 model is trained with helmet dataset. This makes the detection of non-helmet riders easier. Open ALPR is used for the recognition of the license plate [13].The approach mentioned in this paper is applied mainly for power substation .Here for noise elimination mean filtering operation is performed. Background subtraction based on KNN is used to detect the moving objects. Color-based hybrid descriptor is used for feature extraction of the head image segments. SVM classifier is used to classify the head image segments. HOG descriptors are used to identify the pedestrian image segments [21].

License plate recognition is done using image segmentation. Using recognition models like SSD images were segmented and trained by incorporating Inception-V3 and mobile Nets. The characters were segmented and trained by using the above techniques mentioned. Using the trained model license plate was tested. [7].

In this paper, the pedestrians with safety helmet are detected. Here surveillance video images are used. To detect the moving objects, background information is used as valuable prior knowledge. Here single frame is compared with 
background image. Here different distance in pixels is calculated to determine the moving objects. C4 pedestrian detection algorithm is used to accurately detect pedestrians and it is executed on the surrounding of motion objects rather than all pixels of raw frame. The color space transformation and color feature discrimination is used for the safety helmet detection [14].

The non-helmet riders are recognized using video surveillance. Background subtraction is done to eliminate the pedestrians and other entities. Trained model is used to classify the objects. COCO model is used to classify the objects. Using web scraping, different types of helmets were identified. When the riders are carrying their helmet on the body of bike and not wearing them a human face labeled as person was detected. Optical Character Recognition using tesseract is used to detect the license plate of the bike [15].

To get high accuracy of classification they have used transfer learning on top of the CNN module, canny edge detection algorithm is used to obtain appropriately segmented moving objects in each frame and to extract complete moving objects morphological operations are applied. Only when the vehicles reach a specific horizontal line, they are detected. Upper onethird portion of the original image is used to obtain the head portion. To notice the number plates, the motorcyclist image is first converted to gray scale and then the image is threshold. After this, the binary image obtained is inverted. Minimum area rectangles are generated around the contours [16].

HOG descriptors are used for motorcycle detection. For helmet and non-helmet classification, the ROI (i.e. Head region) is passed through a CNN. Haar cascade detector is used for the extraction of license plate numbers. In this paper, they have designed a system in which the motorcyclist's license plate and a short video clip of the violating motorcyclist are automatically uploaded to an automated ticketing systems through which police operators issues a notice to the violators of helmet [20].

\section{DISCUSSIONS}

After studying different research articles, one can observe that the accuracy of the experiment obtained in each of the paper differs because of the methodologies employed and on an average the accuracy rate is around $92 \%$ which is good. The below table 1 shows the individual accuracy obtained in the research articles.
Table 1:Individual accuracy obtained in the research articles.

\begin{tabular}{|c|c|}
\hline Title of the paper and Author nan & Accuracy \\
\hline $\begin{array}{l}\text { Safety Helmet Wearing Detection Based } \\
\text { on Image Processing and Machine } \\
\text { Learning } \\
\text { By Jie li, Min Jiang, Kang Li }\end{array}$ & $80.7 \%$ \\
\hline $\begin{array}{l}\text { An Automatic Detection of Helmeted } \\
\text { and Non-helmeted Motorcyclist with } \\
\text { License Plate Extraction using } \\
\text { Convolutional Neural Network } \\
\text { By Jimit Mistry, Aashish K. Misraa }\end{array}$ & $94.70 \%$ \\
\hline $\begin{array}{l}\text { Automatic Number Plate Recognition } \\
\text { for Motorcyclists Riding Without } \\
\text { Helmet } \\
\text { By Yogirai, Kulkami }\end{array}$ & $96.36 \%$ \\
\hline $\begin{array}{l}\text { Helmet Violation Processing Using } \\
\text { Deep Learning } \\
\text { By Dharma rai, K C }\end{array}$ & $97.22 \%$ \\
\hline $\begin{array}{l}\text { Half and Full Helmet Wearing } \\
\text { Detection in Thailand using Haar Like } \\
\text { Feature and Circle Hough Transform on } \\
\text { Image Processing } \\
\text { By PathasuDoungmala }\end{array}$ & $95 \%$ \\
\hline $\begin{array}{l}\text { An intelligent vision-based approach for } \\
\text { helmet identification for work safety } \\
\text { By Haowu }\end{array}$ & $97.9 \%$ \\
\hline $\begin{array}{l}\text { Helmet Presence Classification with } \\
\text { Motorcycle Detection And Tracking } \\
\text { By J.chiverton }\end{array}$ & $85 \%$ \\
\hline $\begin{array}{lr}\text { Kumhom "Motorcyclist's } & \text { Helmet } \\
\text { Wearing Detection Using } & \text { Image } \\
\text { Processing } & \\
\text { By Thepnimit } & \end{array}$ & $77 \%$ \\
\hline $\begin{array}{l}\text { Automatic Detection Of Motorcyclists } \\
\text { without Helmet } \\
\text { By Romueresilva }\end{array}$ & $97.62 \%$ \\
\hline $\begin{array}{l}\text { Automatic Detection of Bike-riders } \\
\text { without Helmet using Surveillance } \\
\text { Videos in Real-time } \\
\text { By KunalDahiya }\end{array}$ & $98.88 \%$ \\
\hline
\end{tabular}

Table 2: classification between motorcycle and non motorcycle

\begin{tabular}{|l|l|}
\hline Title of the project and author & Accuracy \\
\hline Detection of Motorcyclists without & \\
Helmet in Videos using Convolutional & $91.81 \%$ \\
Neural Network & \\
ByC. Vishnu & \\
\hline $\begin{array}{l}\text { Helmet presence classificationwith } \\
\text { Motorcycle Detection And Tracking }\end{array}$ & $85 \%$ \\
ByJ Chiverton & \\
\hline $\begin{array}{l}\text { Automatic Number Plate Recognition } \\
\text { for Motorcyclists Riding Without }\end{array}$ & $99.5 \%$ \\
Helmet & \\
ByYogirai Kullkami & \\
\hline
\end{tabular}




\section{CONCLUSION}

In this paper, the recent studies in detection of non-helmet motorcycle riders is reviewed. They are characterized and described with respect to steps, they are as follows: Classifying the obtained images into motorcycle and nonmotorcycle, detecting motorcycles among other vehicles using suitable techniques, next step is training the developed model to classify motorcycle riders with and without helmet in the frame. Finally,the challenges are discussed and provided insights into future research.

\section{REFERENCES}

[1] Rattapoom, Waranusast ;Nannaphat, Bundon ; Vasan, Timtong ; Chainarong, Tangnoi ; and Pattanawadee, Pattanathaburt 2013. Machine Vision Techniques for Motorcycle Safety Helmet Detection. 28th International Conference on Image and Vision Computing New Zealand.

[2] Romuere, Silva ;Kelson, Aires ; Thiago, Santos ; Kalyf, Abdala ; Rodrigo, Veras ; and André, Soares 2013. Automatic detection of motorcyclists without helmet. 2013 XXXIX Latin America Computing Conference (CLEI).

[3] Romuere, Silva ;Kelson, Aires ; and Rodrigo, Veras 2014. Helmet Detection on Motorcyclists Using Image Descriptors and Classifiers. 2014 27th SIBGRAPI Conference on Graphics, Patterns and Images.

[4] Shoeb, Ahmed, Shabbeer ; and Merin, Meleet 2017. Smart Helmet for Accident Detection and Notification. 2nd IEEE International Conference on Computational Systems and Information Technology for Sustainable Solutions.

[5] Thepnimit, Marayatr ; and Pinit, Kumhom 2014. Motorcyclist's Helmet Wearing Detection Using Image Processing. Advanced Materials Research Vols 931-932 (2014) pp 588-592.

[6] C. Vishnu ; Dinesh, Singh ; C. Krishna, Mohan ; and Sobhan, Babu 2017. Detection of Motorcyclists without Helmet in Videos using Convolutional Neural Network. Conference Paper · November 2017

[7] Wichai, Puarungroj ; and Narong, Boonsirisumpun 2018. Thai License Plate Recognition Based on Deep Learning. 3rd International Conference on Computer Science and Computational Intelligence 2018.

[8] Xinhua JIANG ,Heru XUE, Lina ZHANG , Yanqing ZHOU 2016. A Study of Low-resolution Safety Helmet Image Recognition Combining Statistical Features with Artificial Neural Network. International Journal of. Simulation

[9] Abhijeet, S. Talaulikar ; Sanjay, Sanathanan ; and Chirag, N. Modi 2019. An Enhanced Approach for Detecting Helmet on Motorcyclists Using Image Processing and Machine Learning Techniques.

[10] Abu, H.M. Rubaiyat ;Tanjin, T. Toma ; Masoumeh, Kalantari-Khandani ; Syed, A. Rahman ; Lingwei, Chen ; Yanfang, Ye ; and Christopher, S. Pan 2016. Automatic Detection of Helmet Uses for Construction Safety. 2016 IEEE/WIC/ACM International Conference on Web Intelligence Workshops.
[11] Amir, Mukhtar ; and Tong, Boon, Tang 2015Vision Based Motorcycle Detection using HOG features. 2015 IEEE International Conference on Signal and Image $g$ Applications (ICSIPA).

[12] Jie, Li ;Huanming, Liu ; Tianzheng, Wang ; Min,

[13] ; Kang, Li ; and Xiaoguang, Zhao 2017. Safety Helmet Wearing Detection Based on Image Processing and Machine Learning. 2017 Ninth International Conference on Advanced Computational Intelligence (ICACI)

[14] Jimit, Mistry ;Aashish, K. Misraa ; Meenu, Agarwal ; Ayushi, Vyas ; Vishal, M. Chudasama ; and Kishor, P. Upla 2017. An Automatic Detection of Helmeted and Non-helmeted Motorcyclist with License Plate Extraction using Convolutional Neural Network.

[15] Kang, Li ;Xiaoguang, Zhao ; Jiang, Bian ; and Min, Tan 2018. Automatic Safety Helmet Wearing Detection.

[16] Kavyashree, Devadiga ; Pratik, Khanapurkar ; Shreya, Joshi ; Shubhankar, Deshpande ; and Yash, Gujarathi 2018. Real Time Automatic Helmet Detection of Bike Riders. IJIRST -International Journal for Innovative Research in Science \& Technology| Volume 4 | Issue $11 \mid$ April 2018.

[17] Yogiraj, Kulkarni ;Shubhangi, Bodkhe ;Amit, Kamthe ; andArchana, Patil 2018. Automatic Number Plate Recognition for Motorcyclists Riding Without Helmet.Proceeding of 2018 IEEE International Conference on Current Trends toward Converging Technologies, Coimbatore, India.

[18] Kunal, Dahiya ; Dinesh, Singh ; and C. Krishna, Mohan 2016.Automatic Detection of Bike-riders without Helmet using Surveillance Videos in Real-time. 2016 International Joint Conference on Neural Networks (IJCNN).

[19] Maharsh, Desai ;Shubham, Khandelwal ; Lokneesh, Singh ; and Prof. Shilpa, Gite 2016. Automatic Helmet Detection on Public Roads. International Journal of Engineering Trends and Technology (IJETT) - Volume 35 Number 5- May 2016.

[20] Pathasu, Doungmala ; and Katanyoo, Klubsuwan 2016. Half and Full Helmet Wearing Detection in Thailand using Haar Like Feature and Circle Hough Transform on Image Processing. 2016 IEEE International Conference on Computer and Information Technology.

[21] Dharma, Raj, K.C. ;Aphinya, Chairat ; Vasan, Timtong ; Matthew, N. Dailey ; and Mongkol, Ekpanyapong 2018 Helmet Violation Processing Using Deep Learning.2018 International Workshop on Advanced Image Technology (IWAIT).

[22] Hao, Wu ; and Jinsong, Zhao 2018. An intelligent vision-based approach for helmet identification for work safety. Volume 100,September 2018 Pages 2016-2017. Elsevier.

[23] J. Chiverton 2012. Helmet presence classification with motorcycle detection and tracking.IET Intell. Transp. Syst., 2012, Vol. 6, Iss. 3, pg No. 259-269. 